\title{
Reflets
}

Revue ontaroise d'intervention sociale et communautaire

\section{L'état de santé des personnes âgées francophones en Ontario} Jocelyne McKellar

Volume 5, numéro 2, automne 1999

La santé des francophones de l’Ontario

URI : https://id.erudit.org/iderudit/026290ar

DOI : https://doi.org/10.7202/026290ar

Aller au sommaire du numéro

Éditeur(s)

Reflets : Revue ontaroise d'intervention sociale et communautaire

ISSN

1203-4576 (imprimé)

1712-8498 (numérique)

Découvrir la revue

Citer cet article

McKellar, J. (1999). L'état de santé des personnes âgées francophones en Ontario. Reflets, 5(2), 290-306. https://doi.org/10.7202/026290ar

Tous droits réservés (C) Reflets : Revue ontaroise d'intervention sociale et communautaire, 1999
Ce document est protégé par la loi sur le droit d'auteur. L'utilisation des services d'Érudit (y compris la reproduction) est assujettie à sa politique d'utilisation que vous pouvez consulter en ligne.

https://apropos.erudit.org/fr/usagers/politique-dutilisation/ 


\section{L'état de santé des personnes âgées francophones en Ontario}

\section{Jocelyne M cK ellar}

C et article présente un ensemble de faits pertinents à la santé et aux habitudes de vie des personnes âgées francophones en O ntario. II examinera entre autres les problèmes de santé, l'activité physique et journalière et le recours au système de soins de santé.

En raison des progrès dans différents secteurs de la médecine et des services sociaux, les personnes ont une espérance de vie beaucoup plus longue qu'elle ne l'était à la fin du siècle dernier (R oberge, B erthelot et Wolfson 1995). M ais en vieillissant, tout individu est confronté à un problème de santé ou à un autre. Les gens âgés sont souvent aux prises avec des problèmes de santé graves qui peuvent occasionner une perte d'autonomie et des problèmes chroniques (R oberge, Berthelot etW olfson 1995:16). C ependant, selon ces mêmes auteurs, l'âge n'est pas le seul facteur qui ait un lien avec l'état de santé. Le sexe, un niveau de scolarité peu élevé, un faible revenu, un travail peu ou non spécialisé, l'absence d'accès à un réseau de soutien informel, des habitudes de vie ou des comportements dits à risques affectent également la santé d'une personne. À 65 ans, les effets cumulatifs de ces facteurs sur la santé et les expériences passées se traduisent par des différences marquées à plusieurs égards entre les hommes et les femmes.

La santé n'est pas simplement l'absence de maladie, mais bien un état de bien-être à la fois physique, mental et social (Andrew et al 1997). La santé est une force fondamentale et dynamique de notre vie quotidienne et est influencée par notre 
situation, nos croyances, notre culture et notre milieu social, économique et physique (Epp 1986:3). L'état de santé de I'homme et de la femme présentera donc des différences tant à un niveau subjectif (auto-évaluation) qu'à un niveau objectif (évalué par un professionnel de la santé).

Cet article s'intéresse particulièrement à l'état de santé subjectif des personnes âgées francophones en 0 ntario. II s'inscrit dans le prolongement des livres de Andrew et al, C onditions de possibilités des services de santé et des services sociaux en français en 0 ntario : un enjeu pour les femmes et de Boudreau et Farmer Profil épidémiologique des francophones de l' 0 ntario au niveau de la santé et du mieux-être: les faits saillants revisités et comparés II en reprend les données qui portent sur la langue et I'âge. Il énonce les faits saillants de l'état de santé des 0 ntariens et des 0 ntariennes, les risques qu'ils prennent volontairement à l'égard de leur santé et l'usage qu'ils font des services de santé.

À cette fin, nous avons utilisé les données relatives aux francophones âgés de plus de 65 ans. La taille de l'échantillon de l'Enquête sur la santé en 0 ntario est de 61000 personnes. U ne procédure de pondération a été employée afin que les chiffres soient représentatifs de la population totale de I'O ntario. $D$ ans l'échantillonnage, 9,4\% des francophones sont âgés de 65 ans et plus (Boudreau et Farmer 1997:4). Par contre, les données disponibles sont limitées. Ainsi, I'utilisation de la variable contrôle «âge », en raison du trop petit nombre de cas, n'a pu être employée lors de l'analyse à plusieurs variables dont le but premier est de comparer les variables liées à la santé entre les francophones et les anglophones. 
F igure 1 - Nombre de problèmes de santé (NPS) par âge et par sexe chez les francophones de l'Ontario, pendant les 12 derniers mois précédents l'enquête, 1990

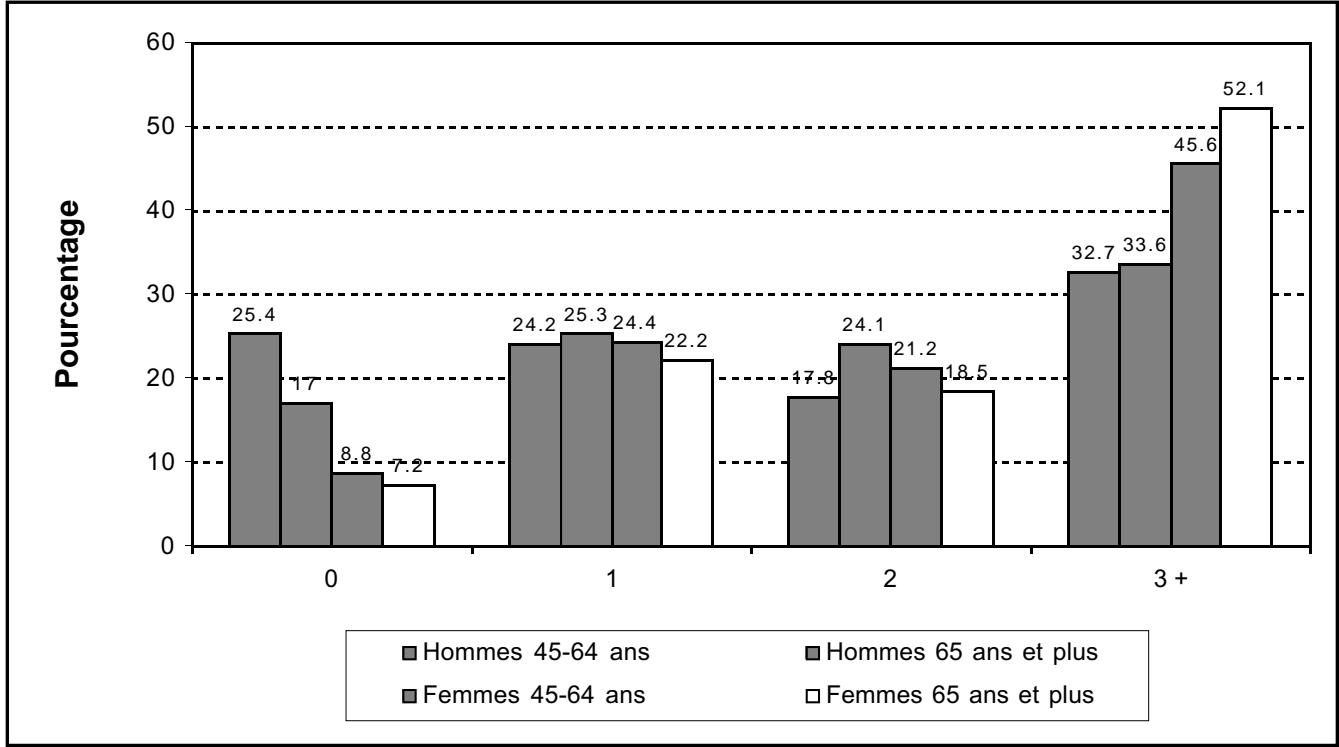

Faits saillants de la figure 1

- Les données montrent qu'avec l'âge, les problèmes de santé deviennent multiples et pluschroniques, tant chez leshommes que chez les femmes.

- Chez lesfrancophonesâgés de plus de 65 ans, 7,2 \% desfemmes et $8,8 \%$ des hommes seulement signalent n'avoir aucun problème de santé contre $17 \%$ et $25,4 \%$ chez les hommes et femmes entre les âges de 45 à 64 ans.

- Environ cinq femmes sur dix âgées de plus de 65 anssignalent trois problèmes de santé ou plus.

- À 65 ans, il y a $12,9 \%$ plus d'hommes qui signalent avoir plus de trois problèmes de santé qu'il y a d'hommes entre 45 et 64 ans. 
Tableau 6.2 - Évaluation de sa propre santé selon les francophones de plus de 65 ans.

\begin{tabular}{|l|r|r|}
\hline & $\begin{array}{c}\text { Évaluation de sa } \\
\text { propre santé }\end{array}$ & Pourcentage \\
\hline Femmes & M oyenne/ M auvaise & 29,2 \\
& B onne \\
& Très bonne & 38,0 \\
& Excellente & 24,7 \\
& Hommes & M oyenne/ M auvaise \\
& B onne & 32,4 \\
& Très bonne & 30,1 \\
& Excellente & 27,8 \\
& & 9,7 \\
\hline
\end{tabular}

\section{Faits saillants du tableau 1}

- II y a légèrement plus d'hommes francophones $(9,7 \%)$ que de femmes (8,1 \%), âgés de 65 et plus qui considèrent que leur santé est excellente.

- II n'en demeure pas moins qu'il y a plus d'hommes (32,4\%) que de femmes $(29,2 \%)$ de 65 ans et plus qui déclarent que leur santé est moyenne ou mauvaise.

\section{La santé des hommes et celle des femmes}

Tel que précisé, un grand nombre de francophones considèrent que leur santé est soit très bonne ou excellente. Toutefois, au fur et à mesure que les individus vieillissent, cette perception positive de l'état de leur santé est sensiblement modifiée chez l'ensemble des $C$ anadiens (Santé nationale et Bien-être social 1993:55). O r, à quoi attribuer le fait que les femmes déclarent plus de problèmes de santé que les hommes et, en même temps, font une meilleure évaluation de leur propre santé que leurs congénères masculins? S'agit-il d'une question de perception, de construction ou d'interprétation divergente de ce que constitue une santé 
«normale » entre les hommes et les femmes? (A ndrew et al 1997:46). Ici, il faut faire attention à l'interprétation de ces données dans la mesure où :

la construction ou la conception de sa propre réalité au niveau de la santé dépend souvent des attentes personnelles des expériences passées avec la maladie, des références et des conditionnements culturels, de œe qu'une personne considère comme étant la normalité et de ce que cette personne voit autour d'elle chez les autres (B oudreau et Farmer 1997:18).

À l'instar de plusieurs auteurs (G ee et Kimball 1987; G uyon1996; Stuckelberger et Hopflinger 1996; Andrew et al 1997), les hypothèses explicatives reposent sur les axes de socialisation de rôles différenciés. Par exemple, on dira que les femmes ont une plus grande tendance à admettre, à rapporter des problèmes de santé et à réagir à ces symptômes. Cette tendance découlerait donc du système de socialisation ou d'attentes sociétales et culturelles propres aux hommes et aux femmes. II serait socialement accepté que les femmes admettent qu'elles sont malades. "Women only appear to be sicker as a result of social factors that allow them to perceive and report illness and to take on the sick role » (Gee et Kimball 1987:34). D ansle même ordre d'idée, Guyon dit que :

les femmes semblent manifester une plus grande sensibilité aux symptômes physiques et psychologiques C ette sensibilité les rendrait plus aptes à percevoir et à reconnaître les sensations physiques comme des signes de maladie, ce qui se traduirait également par une plus grande propension à rechercher de l'aide et à poser des gestes en vue de récupérer la santé, et ce, de façon plus précoce que les hommes (1996: 67).

De plus, en tenant compte de l'espérance plus longue de vie des femmes, elles sont davantage exposées au risque de vivre avec une incapacité que les hommes et d'avoir plus de problèmes de santé. C ependant, Guyon précise que l'état de santé des femmes serait également attribuable à la baisse de leurs revenus et à une 
solitude qui grandit à mesure que les témoins de leur génération disparaissent (1996: 203).

\section{L'impact du statut socio-économique sur la santé}

Il a été démontré maintes fois que le statut socio-économique d'une personne est étroitement lié à la santé (C CN TA 1996; Stuckelberger et $\mathrm{H}$ opflinger 1996; R oberge, Berthel ot etWolfson 1995; Santé C anada 1995; Paquet 1989; 1994; Guyon et al 1996). C es auteurs s'entendent pour dire que les personnes faisant partie de milieux socio-économiques défavorisés sont davantage confrontées à une détérioration précoce de la santé. D e surcroît, les gens à faible revenu risquent davantage de mourir par suite de chutes accidentelles, de maladies respiratoires, de pneumonie et de cirrhoses du foie. Il s sont davantage exposés à certaines maladies comme les troubles mentaux, l'hypertension et les affections articulaires, en plus d'être exposés à certains risques tels le tabac, l'alcool et la sédentarité (Paquet 1989: 21).

N os analyses de l'ensemble desindicateurs socio-économiques montrent que les personnes âgées franco-ontariennes ont connu des conditions de vie défavorables. De plus, l'absence de sécurité d'emploi, d'avantagessociaux et l'impossibilité de bénéficier d'autres formes de revenusà cause d'une base salariale insuffisante, forment un ensemble qui caractérise davantage la réalité des femmes âgées que celles des hommes âgés (G uyon 1996: 204). La discrimination présente tout au long de la vie des femmes francophones peut être associée au fait que ces dernières signalent un état de santé moins bon que celui des hommes. Andrew et al confirment que:

I'évaluation de son propre état de santé varie non seulement selon les revenus du ménage, mais aussi selon le niveau d'éducation des répondantes et répondants. [... ] A insi, les femmes francophones âgées de plus de 65 ans, dont le revenu et le niveau d'éducation sont inférieurs aux hommes, vivraient, en cequi a trait à leur santé, les conséquences d'une qualité de vie et d'une condition socio-économique depuis longtemps défavorisées (1997: 46). 
En effet, l'association entre la mauvaise santé et la pauvreté est particulièrement forte chez les femmes âgées (G uyon 1996: 205). Bien que Guyon parle des Q uébécoises, il n'est pas surprenant de constater que plus de la moitié des femmes francophones de plus de 65 ans s'estiment en moyenne ou mauvaise santé.

Plusieurs études ont confirmé que l'exposition répétée à des événementstraumatisantsou des grandsmoments de crise familiale risquent d'affecter le bien-être mental et physique d'un individu. Le stress associé à la mort ou à la prise en charge d'une personne malade a aussi un effet négatif sur la santé (G ee et K imball 1987; Santé et Bien-être social 1993).

Lesfemmes sont parmi les plus grandes pourvoyeuses de soins auprès d'un conjoint malade ou d'un parent. La plupart des soignantes sont elles-mêmes des personnes âgées de plus de 65 ans. La prise en charge d'une personne âgée entraîne parfois des conséquences comme la dépression, la détresse psychologique, une baisse de satisfaction face à la vie, des conflits interpersonnels, I'isolement social et des problèmes physiques liés au stress (Santé C anada 1997-1998: 6). C ette prise en charge risque de nuire à la santé et de limiter leur capacité à fournir le soutien essentiel aux êtres qui leurs sont chers (Santé C anada 1997-1998: 7).

Ainsi, lesliens sociaux exercent un effet protecteur sur la santé (Guyon et al 1996: 210). Des recherches effectuées confirment que l'absence d'aide, d'appui ou de soutien au plan familial, social ou communautaire constitue un facteur de risque pour la santé (C happell 1996: 62). Par exemple, une étude de Santé C anada révèle que les hommes qui ont moins de contacts sociaux ont deux fois plus tendance à mourir prématurément que ceux qui en ont davantage (1995: 3). Les données sur le degré de soutien social desfrancophones de plus de 65 ansindiquent qu'ilsjouissent d'un soutien social faible ou moyen, mais que les hommes ne meurent pas prématurément. C ependant, il est fort possible que cette absence de liens sociaux affecte leur santé, ce que montrent certains résultats. 


\section{Les habitudes et les styles de vie}

Le genre d'activités, de pratiques ou de comportementsindividuels de santé auxquelles les personnes âgées peuvent s'adonner, à l'extérieur ou à domicile, peuvent à plus ou moins long terme influencer leur état de santé et leur qualité de vie (Andrew et al 1997; Santé et Bien-être social 1993). Par style de vie, nous entendonsl'ensemble des comportements individuelsqui peuvent contribuer ou nuire à la santé ( $p$. ex. surmédicalisation, sédentarité, mauvaise alimentation).

Il est intéressant de constater que certaines habitudes sont acquisesà une époque où leurs méfaitsn'étaient pas encore connus (Stuckelberger et H opflinger 1996: 47). À vrai dire, les valeurs et les méthodes d'éducation préconisées lorsque les personnesâgées étaient enfants, diffèrent de celles des générations suivantes (C happell 1996: 62). D onc, comme l'énoncent Stuckelberger et $\mathrm{H}$ opflinger, c'est souvent plus tard qu'on découvre les risques élevés de décès liés à certaines habitudes de vie (1996: 47).

À titre d'exemple, la notion de prévention «traditionnelle »a longtemps été associée à la prévention des maladies contagieuses et au dépistage précoce des maladies. A ujourd'hui nous parlons plutôt de la réduction des risques liés à l'environnement social et économique (Berger et M aillous-Poirier 1993: 146). De plus, aujourd'hui, la transmission de l'information se fait beaucoup plus facilement et rapidement, bien qu'il soit possible que cette information ne soit pas accessible aux personnes âgées francoontariennesen raison de leur sous- scolarisation, de l'indisponibilité de la documentation en français et du mode «traditionnel » de transmission des savoirs.

Les résultats d'enquêtes récentes sur la santé montrent que les adultes vivants dans des ménages à faible revenu, lorsqu'on les compare aux ménages ayant des revenus moyen et supérieur, ont des chances plus élevées d'avoir des comportements présentant des risques pour la santé (Santé C anada 1995: 19). Par exemple, il a été démontré que les individus de milieux socio-économiques 
défavorisés sont plus nombreux à consommer quotidiennement du tabac, des tranquillisants ou des somnifères. Ils étaient aussi moins nombreux à connaître certaines des causes des maladies cardiaques et des méthodes de prévention des maladies (Santé C anada 1995: 19).

Examinons ce que nous disent les données sur certaines habitudes et styles de vie des personnes âgées franco- ontariennes. Sont-elles plus à risque que la population non-francophone?

\section{Les habitudes alimentaires et l'activité physique}

Les habitudes alimentaires des hommes francophones diffèrent de celles des femmes francophones. Les femmesfrancophones de 45 à 64 ans consomment moins de matières grasses que les hommes francophones (Andrew et al. 1997: 61). Est-ce que les hommes mangent en dehors du foyer plus souvent, en particulier dans des établissements de restauration rapide (Andrew et al. 1997: 61)? Q uelles sont les autres raisons possibles pouvant expliquer ces mauvaises habitudes al imentaires?

La scolarité et le statut économique sont les deux principaux facteurs qui affectent la capacité d'une personne à adopter un régime alimentaire sain. D'après C ousins (1996: 67), un statut socio-économique inférieur peut être associé à une mauvaise nutrition. À vrai dire, cen'est pas l'argent en soi qui est important pour la santé, mais lesconditions, les possibilités et les commodités qu'il offre (C happell 1996:63).Ainsi, l'argent permet à un individu de s'acheter de la nourriture qui lui permet une bonne diète. L'absence d'information adaptée en français, en raison du haut taux de sous-scolarisation de la population étudiée, peut également avoir une importance sur la capacité de maintenir une diète saine.

L'activité physique est importante à tous les âges. Pour les personnesâgées, l'activité physique aide au maintien de la mobilité, de la flexibilité et de l'autonomie. L'étude de Paquet (1989) constate que I'activité physique protège contre la maladie coronarienne.

Selon les données de l'E nquête sur la santé en 0 ntario (ESO 1990), $29 \%$ des femmes et $46 \%$ des hommes francophones âgés 
de plus de 65 ans font régulièrement de l'exercice. Q uel que soit le groupe d'âge, les femmes font rarement de l'activité physique. À quoi pouvons-nous attribuer cet écart entre les sexes? $R$ elèvet-il d'une perception ou d'une interprétation de ce qu'est l'activité physique ou encore, du degré d'activité? Pourquoi les femmes doivent-elles limiter ainsi leurs activités?

D 'une part, les femmes sont davantage alitées ou sont dans I'incapacité de fonctionner normalement (Boudreau et Farmer 1997 :19), ce qui limite d'autant leur possibilité de s'engager dans certaines activités physiques. D 'autre part, les rôles multiples des femmes les empêchent parfois de faire des sorties ou de faire de l'activité physique. En plus des tâches domestiques, les femmes âgées doivent parfois s'occuper d'un conjoint ou d'un parent malade et consacrent une grande partie de leur temps aux soins des autres. Cette prise en charge volontaire peut expliquer pourquoi les femmes font rarement de l'activité physique.

\section{Les routines professionnelles dangereuses}

Tableau 2 - Les routines professionnelles dangereuses, des francophones et des anglophones ontariens de plus de 65 ans, selon le sexe.

\begin{tabular}{|l|r|r|r|r|}
\hline & \multicolumn{2}{|c|}{ Francophones } & \multicolumn{2}{c|}{ Anglophones } \\
\hline & Femmes & Hommes & Femmes & Hommes \\
\hline $\begin{array}{l}\text { Routines professionnelles } \\
\text { dangereuses }\end{array}$ & \multicolumn{1}{|c|}{$\%$} & $\%$ & $\%$ & $\%$ \\
\hline Jamais & 27,6 & 26,9 & 39,8 & 38,4 \\
Parfois & 24,8 & 35,9 & 29,7 & 32,3 \\
Souvent & 41,1 & 25,0 & 21,6 & 17,8 \\
Toujours & 6,5 & 12,2 & 8,9 & 11,5 \\
\hline
\end{tabular}

Les routines professionnelles dangereuses au travail peuvent avoir un impact sur la santé des gens au fur et à mesure qu'ils avancent en âge. $D$ 'après les données au tableau 2, il y a plus de femmes francophonesque d'hommes francophonesqui exercent un travail «dangereux » au point de vue de leur santé. Par ailleurs, il y a plus 
de francophones que d'anglophones qui déclarent que leurs routines au travail sont « souvent » ou «toujours » dangereuses. C ette question des routines professionnelles dangereuses est importante dans la mesure où elle rejoint la question des occupations traditionnelles des hommes franco-ontariens.

Comme le préciseA ndrew et al, ceschiffres sont certainement à mettre en rapport avec le style de vie et l'occupation des personnes ayant participé à l'enquête (1997: 56). U n travail dangereux au niveau de la santé, est un travail manuel, soit à l'usine ou à la ferme. $0 \mathrm{n}$ sait que les hommes francophones de plus de 65 ans ont détenu davantage d'emplois dansl'usinage de matières premières, dans les industries minières, des pêches, des forêts et de forage. C es occupations sont souvent monotones, exigent de la force physique et sont parfois dangereuses. Pouvons-nous dèslors conclure que les hommes francophones seront davantage atteints de certains types de troubles physiques?

Les données fournies dans l'E nquête sur la santé en 0 ntario (ESO 1990) sur les affections les plus courantes montrent effectivement que $24,7 \%$ des hommes francophones sont atteints de problèmes liés au système musculo-squelettique et 23,5\% indiquent avoir des problèmes avec leur système respiratoire (Andrew et al. 1997: 42). C es types de problèmes peuvent être le résultat d'un environnement de travail insalubre.

\section{La consommation de médicaments}

La consommation de médicaments est révélatrice d'un mauvais état de santé, mais elle est aussi un facteur de risque pour la santé. Par exemple, la combinaison de drogues peut causer des effets secondaires indésirables ou d'autres problèmes risquant d'affecter la santé comme de la négligence au niveau de l'alimentation ou I'hygiène (AR F 1993:5). Lorsque nous parlons de la consommation de médicaments, il faut également examiner la manière dont les personnes âgées suivent leurs ordonnances. II existe plusieurs raisons pour lesquelles les personnes âgées n'observent pas bien leurs ordonnances:

elles ont fréquemment dela diffiaultéà ouvrir les flacons de médicaments, munis d'un couverde de séarité à 
l'épreuve de la manipulation par les enfants; à cause de leur mauvaise vision, elles lisent mal les étiquettes sur les contenants ou sont incapables d'identifier les pilules correctement ou elles ne savent pas lire; au surplus, elles peuvent aussi oublier ou mal comprendre les directives d'un médean à cause d' une ouïe déficiente (M ishara et $R$ iedel 1984: 225).

Tableau 3 - Consommation de médicaments sur ordonnance au cours des quatre dernières semaines, francophones ontariens, selon le sexe et âge.

\begin{tabular}{|l|r|r|r|}
\hline Âge & $\begin{array}{c}\text { Nombre de } \\
\text { médicaments }\end{array}$ & Femmes & Hommes \\
\hline $\mathbf{4 5}$ à $\mathbf{6 4}$ ans & 0 & $37,5 \%$ & $41,9 \%$ \\
& 1 ou 2 & $40,3 \%$ & $39,2 \%$ \\
& 3 ou 4 & $15,0 \%$ & $10,0 \%$ \\
& 5 ou + & $7,3 \%$ & $8,9 \%$ \\
\hline 65 ans et + & 0 & $14,7 \%$ & $13,8 \%$ \\
& 1 ou 2 & $45,7 \%$ & $47,6 \%$ \\
& 3 ou 4 & $21,5 \%$ & $15,6 \%$ \\
& 5 ou + & $18,0 \%$ & $23 \%$ \\
\hline
\end{tabular}

À partir de l'âge de 65 ans, le nombre d'hommes et de femmes francophones qui ont consommé plus de cinq médicaments différents au cours des quatre dernières semaines précédant l'Enquête, il double. Bien qu'il y ait toujours sensiblement plus d'hommes que de femmes qui ne prennent aucun médicament, on constate que $23 \%$ des hommes et $18 \%$ des femmes de plus de 65 ans consomment plus de cinq médicaments différents (Andrew et al. 1997: 68). Par contre, les données n'indiquent pas la périodicité de la prise des médicaments, s'il s'agit d'une consommation journalière ou occasionnelle, si cette consommation est liée à une dépendance ou non (B oudreau et Farmer 1997: 53). Comme le mentionnent Boudreau et Farmer, les 
résultats sont difficiles à interpréter sans information supplémentaire. U ne étude qualitative sur les attitudes face à I'usage de médicaments en vente libre ou sur ordonnance ai derait dans l'interprétation des données (1997: 54). D e plus, une étude quantitative multivariée permettrait d'en savoir davantage sur la consommation de médicaments selon certains facteurs sociaux, en particulier la difficulté de suivre les ordonnances pour les personnes qui éprouvent des difficultés de lecture.

\section{Les consultations professionnelles}

L'utilisation des services de santé est souvent perçue comme menaçante en raison des changements possibles provoqués par cette consultation. Paquet (1989:67) mentionne que le manque d'information, la gêne, voire la honte de son corps, le manque de perspectives d'avenir constituent un frein à cette utilisation. C ela dit, il existe un lien étroit entre la scolarité et la santé dans la mesure où les gens plus scolarisés utilisent davantage les services de santé parce qu'ils peuvent plusfacilement faire valoir leur droits et connaissent un peu plus les services disponibles (Paquet 1994:231).

Les données fournies par l'E nquête sur la santé en 0 ntario (1990) offrent très peu de renseignements sur les différents obstacles qui nuisent à l'accessibilité aux services de santé. Bien que les données n'indiquent pas l'âge des répondants, nous croyons que de tels obstacles sont présents chez les personnes âgées de plus de 65 ans.

C ela n'empêche pas que les hommes et les femmes francophones de plus de 65 ans ont consulté plus de neuf fois des professionnels de la santé, en 1989. M ais, nous ne savons pas si ces professionnels de la santé offrent des services en français, bilingues ou unilingue anglais. 
Tableau 4 - Nombre de consultations auprès de professionnels de la santé, selon le groupe linguistique et le sexe, personnes âgées de plus de 65 ans, durant la dernière année. O ntario, 1990.

\begin{tabular}{|l|rr|rr|}
\hline \multicolumn{1}{|c|}{$\begin{array}{c}\text { Total de } \\
\text { consultations }\end{array}$} & \multicolumn{2}{|c|}{ Francophones } & \multicolumn{2}{c|}{ Anglophones } \\
Hommes & Femmes & Hommes & Femmes \\
\hline 0 & & & & \\
$1-2$ & $4,9 \%$ & $4,6 \%$ & $5,9 \%$ & $3,5 \%$ \\
$3-5$ & $10,5 \%$ & $9,9 \%$ & $11,5 \%$ & $10,7 \%$ \\
$6-8$ & $19,2 \%$ & $19,5 \%$ & $20,5 \%$ & $22,3 \%$ \\
$9+$ & $12,8 \%$ & $20,7 \%$ & $16,7 \%$ & $16,4 \%$ \\
& $52,5 \%$ & $45,3 \%$ & $45,4 \%$ & $47,0 \%$ \\
\hline
\end{tabular}

\section{Faits saillants du tableau 4}

- Si, en général, les femmes consultent davantage les professionnels de la santé durant leur vie, à partir de 65 ans, ce sont les hommes qui sont les plus nombreux $(52,5 \%)$ à consulter soit plus de neuf fois par année.

- Les hommes francophones de plus de 65 ans (52,5\%) ont plus de contact avec un professionnel de la santé que les hommes anglophones du même groupe d'âge (45,4\%).

Les résultats de l'enquête concernant I'hospitalisation démontrent que celle-ci est plus commune chez les hommes francophones de plus de 65 ans (24,1\%) que chez les femmes (14,7\%). C ette réalité s'applique également à l'ensemble de la population canadienne.Ainsi,

les hommes âgés sont plus souvent hospitalisés que les femmes pour des cancers, des maladies de l'appareil circulatoire, de celles de l'appareil respiratoire, de I'appareil digestif et celles des organes génito-urinaires. Par contre, les femmes vont plus souvent à l'hôpital pour des maladies du système ostéo-musculaire et pour des causes dites "externes », c'est-à-dire pour des accidents, des empoissonnements et des traumatismes dus à la violence (D umas et D esjardins 1993: 94). 
Q ue les taux de séjours hospitaliers masculins soient supérieurs aux taux fémininsapparait paradoxal, compte tenu desobservations faites sur la prévalence des problèmes de santé chez les hommes et les femmes (D umas 1993: 95). Les femmes francophones de plus de 65 ans se déclarent plus que les hommes francophones en mauvaise santé, mais sont moins souvent hospitalisées et consultent moins un professionnel de la santé. C eci pourrait être attribué aux différences quant à la nature des problèmes de santé des hommes et des femmes ou même aux différences dans les styles de vie et d'occupations. Le mode de vie des hommes, leur faible réseau social, leur niveau de scolarité moins prolongé et leur routines professionnelles dangereuses sont, sans doute, autant de facteurs de risques pour les hommes et permettent de comprendre davantage pourquoi les hommes sont plus vulnérables et ont un état de santé si fragile à la vieillesse. $D$ 'après les données présentées, nous pouvons conclure que les hommes francophones de plus de 65 ans sont davantage à risque d'avoir une santé précaire comparativement aux hommes anglophones et aux femmes francophones.

\section{Conclusion}

Les données présentées mesurent la perception de la santé des personnes âgées franco-ontariennes. Bien que le vieillissement engendre une détérioration de la santé, cette dernière dépend beaucoup des conditions de vie actuelles et passées. Cette détérioration de la santé engendre un niveau d'activité limité, la consommation de plusieurs médicaments et les maladies ayant, comme conséquences, I'utilisation plus fréquente aux professionnels de la santé et dans les cas sérieux, I'hospitalisation.

$\mathrm{N}$ ous voyons à quel point le fait d'être homme ou femme, francophone ou anglophone, détermine une chaîne d'événements physiologiques, familiaux, sociaux et économiques différenciés qui se répercutent sur le vieillissement et la santé de la personne. 
Q uoi qu'il en soit, à 65 ans, les effets cumulatifs des habitudes et des expériences de vie antérieures ont des effets marqués sur la santé (Santé C anada 1995).

Les études démontrent que les plus pauvres et les moins scolarisés cumulent les plus mauvaises habitudes de vie, la plus forte consommation de médicaments (p. ex. les tranquillisants) et le plus grand nombre de problèmes de santé, et parmi eux, les plusincapacitants (Paquet 1989: 20). Les données sur les personnes âgées franco-ontariennes fournies dans cet article semblent appuyer de telles conclusions.

En effet, nous pouvons établir un parallèle similaire entre les conditions économiques difficiles et l'état de santé pour de très nombreuses personnes âgées franco- ontariennes. C omme nous I'avons dit plus haut, l'état de santé varie en fonction des revenus et du niveau d'éducation. $0 \mathrm{r}$, beaucoup de personnes âgées francophones qui ont très peu de scolarité, ont occupé un travail plus dangereux et affichent des revenus plus bas que les non francophones ont des effets plus marqués sur leur l'état de santé. L'état de santé des personnes âgées francophones en 0 ntario semblerait être lié à l'ensemble de ces conditions de vie.

Si un plus grand nombre d'hommes francophones évaluent leur santé comme étant «très bonne » ou même «excellente », les données concernant leur fréquentation du système de santé semblent indiquer le contraire, c'est-à-dire qu'ils ont une santé plus précai re que les femmes francophones à partir de l'âge de 65 ans. Du moins, une telle conclusion s'impose d'elle-même suite aux résultats sur les routines à risque, le nombre de médicaments consommés, le nombre de consultation auprès d'un professionnel de la santé, la nature des problèmes de santé et le taux de séjours hospitaliers.

Par contre, lesfai bles revenus desfemmes semblent aussi affecter leur état de santé. Les femmes francophones âgées de plus de 65 ans déclarent plus de problèmes de santé que les hommes. C ette tendance persiste compte tenu du fait que leur espérance de vie surpasse celle des hommes. Ces problèmes de santé peuvent expliquer pourquoi un grand nombre de femmes francophones s'engagent rarement dans des activités physiques. Bien que les 
femmes francophones aient connu des conditions économiques et sociales plus difficilesque les hommes francophones et qu'elles déclarent plus de problèmes de santé, elles vivent en moyenne jusqu'à un très grand âge.

\section{Bibliographie}

AN DR EW et al. (1997). C onditions de possibilités des services de santé et des services sodaux en français en 0 ntario: un enjeu pour les femmes, 0 ttawa,Table féministe franco phone de concertation provinciale de l'O ntario.

BO UDR EAU, F. et D. FAR MER (1996). Projet santé et services sociaux en milieu francophone ontarien: volet 1. Profil épidémiologiques des francophones de l'ontario au niveau de la santé et du mieux-êrte: les faits saillants revisités et comparés,Toronto, C ollège universitaire G lendon, département de sociologie. 